\title{
SERVIÇOS DE ZOONOSES E O SEU PAPEL NA VIGILÂNCIA EM SAÚDE PARA LEISHMANIOSE VISCERAL
}

Chelsea Pereira de Souza ${ }^{1}$, Patrícia Ferreira da Silva ${ }^{1}$, Matheus de Carvalho Moreno ${ }^{2}$, Lourdes Aparecida Zampieri D’ Andrea ${ }^{1,2}$

${ }^{1}$ Instituto Adolfo Lutz, Centro Laboratório Regional de Presidente Prudente/SP.

${ }^{2}$ Universidade Estadual Paulista, Campus de Presidente Prudente/SP. Email: chelsea souza@hotmail.com

\section{RESUMO}

A leishmaniose visceral (LV) é uma doença zoonótica de grande importância em saúde pública e as ações realizadas pelos Serviços de Zoonoses (SZ) podem minimizar seu transtorno. O objetivo foi avaliar a situação dos Serviços de Zoonose dos municípios na região de saúde de Presidente Prudente/SP e compreender o papel que eles desempenham na vigilância em saúde para leishmaniose visceral. Foi realizado tratamento cartográfico da informação sobre a situação epidemiológica da leishmaniose visceral e o tipo de SZ ofertado nos municípios de estudo. Dos 45 municípios estudados, $62,2 \%$ possuem SZ totalmente estruturado; $6,7 \%$ não possuem estrutura física e funcionam parcialmente; 4,4\% não funcionam e $26,7 \%$ não o possuem. Concluímos que é de grande relevância o papel que os SZs desempenham na vigilância em saúde e há uma elevada quantidade de municípios que não possuem SZ estruturado, fato que pode dificultar no controle da leishmaniose visceral, favorecendo a disseminação na região.

Palavras-chave: leishmaniose visceral, zoonose, serviços de saúde, vigilância epidemiológica, controle de doenças transmissíveis

\section{ZOONOSES SERVICES AND THEIR ROLE IN HEALTH SURVEILLANCE FOR VISCERAL LEISHMANIASIS}

\begin{abstract}
The visceral leishmaniasis (VL) is a zoonotic disease of great importance in public health and the actions performed by Zoonoses Services (ZS) can minimize their disorder. The objective was to evaluate the situation of zoonosis services of the counties in the health region of Presidente Prudente/SP and understand the role its play in health surveillance for visceral leishmaniasis. Was performed cartographic information on the epidemiological situation of the visceral leishmaniasis and the type ZS offered in the counties belonging of study. Of the 45 counties studied, $62.2 \%$ have fully structured ZS; $6.7 \%$ have no physical structure and partially function; $4.4 \%$ do not work and $26.7 \%$ do not possess. We conclude that, is of great relevance, the role played by ZSs in health surveillance and there is a high number of counties that do not have structured ZS, a fact that can make it difficult to control the visceral leishmaniasis, favoring the dissemination in the region.

Keywords: visceral leishmaniasis, zoonoses, health services, epidemiological monitoring, communicable disease control.
\end{abstract}

\section{INTRODUÇÃO}

Segundo a Organização Mundial de Saúde (OMS), zoonoses são definidas como doenças ou infecções naturalmente transmissíveis entre animais vertebrados e seres humanos ${ }^{1}$. A transmissão pode ocorrer de forma direta (contato com secreções ou contato físico através de mordeduras e/ou arranhaduras) ou indireta (vetores, consumo de alimento contaminado com o agente etiológico), entre outras ${ }^{2}$. Em decorrência de sua importância, tanto do ponto de vista social quanto do ponto de vista econômico, é necessária a adoção de medidas capazes de minimizar transtornos através da aplicação de métodos adequados para a prevenção, controle ou erradicação destas doenças ${ }^{3}$. É necessário ainda para que tais medidas sejam adotadas, que se tenha 
conhecimento sobre suas características como a causa, incidência e prevalência ${ }^{4}$.

A primeira atividade relacionada ao controle de zoonoses implantada no Brasil foi a construção, de canis públicos nas principais capitais do país. Posteriormente, já na década de 70, houve a criação dos primeiros Centros de Controle de Zoonose (CCZ), em que as atividades eram voltadas principalmente para controle da raiva ${ }^{5,6}$. Os CCZs são instituições municipais ligadas a órgãos locais de saúde, podendo possuir estrutura física e personalidade jurídica legalmente estabelecida. Suas competências e atribuições são voltadas para os programas de controle de zoonoses, de doenças transmitidas por vetores e de agravos provocados por animais peçonhentos ${ }^{7,8}$. O dimensionamento do tipo de modalidade de CCZ ou de canil municipal a ser implantado nos municípios é feito de acordo com a população humana existente daquela localidade ${ }^{9}$.

A Portaria do Ministério da Saúde MS/GM no 1.138 , de 23 de maio de $2014^{10}$ definiu as ações e os serviços de saúde voltados para vigilância, prevenção e controle de zoonoses e de acidentes causados por animais peçonhentos e venenosos, de relevância para a saúde pública. Foram publicadas pelo MS, normas técnicas específicas ao assunto. Em conjunto com a Portaria no 758, de 26 de agosto de 2014, as atribuições relacionadas ao controle de zoonoses foram mais bem definidas e o CCZ passou a ser substituído pela Unidade de Vigilância de Zoonoses (UVZs) ${ }^{11}$. Essa mudança possibilitou que as ações fossem mais direcionadas, trazendo melhorias para o serviço ${ }^{6}$.

As ações, atividades e estratégias de vigilância de zoonoses visam eliminar ou diminuir os riscos de transmissão à população humana. Para isso, é necessário o levantamento do impacto que o tipo de zoonose causa na saúde pública, através da avaliação da gravidade, capacidade de disseminação, instalação e transmissão, a população vulnerável, as espécies animais envolvidas, o tempo e a área onde ocorre a doença ${ }^{12,13}$.

A leishmaniose visceral (LV) é uma doença crônica e sistêmica causada pelo protozoário tripanosomatideo, Leishmania infantum (sinonímia Leishmania (Leishmania) (chagasi $)^{14}$. Cerca de $90 \%$ do total de casos ocorrem em grupo de seis países, do qual o Brasil faz parte ${ }^{15,16}$. A doença é considerada uma zoonose, e a forma de transmissão ocorrem pela picada da fêmea de vetores flebotomíneos infectadas, tendo como a principal espécie transmissora o Lutzomyia longipalpis ${ }^{13}$. No ambiente urbano, o cão é considerado reservatório de maior importância ${ }^{17,18}$. Quando acomete o humano, os sintomas mais comuns incluem esplenomegalia, pancitopenia, febre e perda de peso, podendo levar o indivíduo a morte quando não diagnosticada e tratada adequadamente ${ }^{19,20}$.

O MS estabeleceu em 2006 o Programa de Vigilância e Controle da Leishmaniose Visceral (PVCLV), tendo como objetivo a diminuição de morbidade, letalidade assim como a dispersão territorial da doença ${ }^{12}$. O programa estabelece como medidas o diagnóstico e o tratamento precoce dos casos em humano, diminuição dos vetores (flebotomíneos) e controle dos reservatórios domésticos ${ }^{12,21,22}$.

As recomendações a cerca das ações de vigilância em saúde que devem ser realizadas para leishmaniose visceral varia de acordo com a condição epidemiológica do município e a transmissibilidade da doença. Os municípios podem ser classificados como silenciosos, em que não possuem casos autóctones da doença, municípios em transmissão onde possuem registro de casos notificados, podendo ser ainda de transmissão esporádica moderada ou intensa, conforme o número de casos ocorridos no período avaliado ${ }^{5,12}$. Diante do exposto, é de extrema importância a presença de um Serviço de Zoonoses ou CCZs estruturado e funcionando de forma adequada como um fator primordial no que tange as ações de vigilância e controle da LV numa dada localidade. Entende-se como Serviço de Zoonose, atividades recomendadas pelo PVCLV e que o município deve realizar em relação ao reservatório canino.

O estudo teve como principal objetivo avaliar a situação dos Serviços de Zoonose ou Centros de Controle de Zoonoses dos municípios na região de saúde de Presidente Prudente e compreender o papel que eles desempenham na vigilância em saúde para leishmaniose visceral.

\section{METODOLOGIA}

A área de estudo abrange 45 municípios pertencentes à Rede Regional de Atenção à Saúde (RRAS) 11 de Presidente Prudente, localizada na macrorregião de Centro-Oeste do estado de São Paulo, Brasil. 
Os dados levantados sobre a situação epidemiológica e a disponibilidade de Serviços de Zoonoses ou CCZs dos municípios, foi considerada como base o período de Maio de 2018. Os mesmos foram classificados epidemiologicamente em: município em investigação; município em transmissão humana e canina para leishmaniose visceral; município em transmissão canina para leishmaniose visceral; município silencioso receptivo vulnerável; município silencioso não receptivo vulnerável e município com notificação de casos caninos, não receptivos. Com relação à disponibilidade de Serviço de Zoonoses ou CCZ ofertado, foram classificados em: totalmente estruturado; sem estrutura física e parcialmente funcionando; sem estrutura física e sem funcionamento e sem nenhum tipo de Serviço de Zoonoses.

A fonte de dados utilizados foi fornecida pelo Laboratório de Parasitologia do Centro de Laboratório Regional - Instituto Adolfo Lutz de Presidente Prudente - V (CLR- IAL- PP V). Para o tratamento cartográfico da informação, os dados coletados foram transpostos em tabelas de informação gráfica para geração de um Sistema de Informações Geográficas (SIG). Foram utilizadas bases de dados cartográficos disponibilizadas pelo Instituto Brasileiro de Geografia e Estatística (IBGE), os dados coletados foram convertidos em mapas temáticos, analisados por superfícies de interpolação através do software Arc Giz 10.3 do laboratório de Biogeografia da Universidade Estadual Paulista (UNESP) "Júlio de Mesquita Filho", Campus de Presidente Prudente. A situação epidemiológica do município foi demonstrada em mapa temático coroplético e o Serviço de Zoonoses expresso através de símbolos (pontos), com respectiva legenda explicativa.

Quanto aos aspectos éticos, os dados aqui apresentados, fazem parte de resultados parciais obtidos com $\mathrm{o}$ um estudo maior denominado Leishmaniose visceral na região de Presidente Prudente, São Paulo: distribuição espacial e rotas de dispersão, avaliado e aprovado pelo Comitê Técnico Científico do Instituto Adolfo Lutz - CTC-IAL - 25H.2015, aprovado pelo Comitê de Ética em Pesquisa do IAL - CEPIAL, CAAE: 53247716.8.0000.0059, parecer №. 1.934.175 e pela Comissão de ética no Uso de Animais - CEUA- IAL -02/2016.

\section{RESULTADOS}

Dos 45 municípios que compõem a região de saúde de Presidente Prudente, 62,2\% (28) possuem Serviço de Zoonoses totalmente estruturado, sendo eles: Alfredo Marcondes, Álvares Machado, Caiuá, Dracena, Emilianópolis, Flora Rica, Irapuru, Junqueirópolis, Marabá Paulista, Martinópolis, Monte Castelo, Nova Guataporanga, Ouro Verde, Panorama, Paulicéia, Piquerobí, Presidente Epitácio, Presidente Prudente, Presidente Venceslau, Quatá, Rancharia, Ribeirão dos Índios, Santa Mercedes, Santo Anastácio, Santo Expedito, São João do Pau d'Alho, Teodoro Sampaio e Tupi Paulista (Figura 1 e Tabela 1). Destes, 15,6\% (sete), Alfredo Marcondes, Álvares Machado, Cauiá, Martinópolis, Piquerobi, Rancharia, Ribeirão dos Índios possuem apenas transmissão canina e 4,4\% (dois) Quatá e Emilianópolis encontram-se em investigação (Figura 1 e Tabela 1). Os demais, $42,2 \%$ (19) possuem transmissão humana e canina para leishmaniose visceral.

$\mathrm{Na}$ Figura 1 estão apresentadas a situação epidemiológica da leishmaniose visceral e a disponibilidade de Serviços de Zoonoses nos municípios que fazem parte da região de saúde de Presidente Prudente/SP, no período de maio de 2018.

$\mathrm{Na}$ tabela 1 estão relacionados os 45 municípios da região de saúde de Presidente Prudente/SP, classificados em relação ao tipo de Serviço de Zoonose existente em seu território e a situação epidemiológica quanto a leishmaniose visceral em de maio de 2018.

Em 6,7\% (três) dos municípios analisados (João Ramalho, Presidente Bernardes, Mirante do Paranapanema), o Serviço de Zoonoses não possui estrutura física própria e funciona apenas parcialmente (Tabela 1). Coletam as amostras biológicas dos cães, mas realizam a triagem sorológica com teste rápido (TR) DPP BioManguinhos para leishmaniose visceral canina no laboratório de referencia regional para esse agravo, que é o IAL de Presidente Prudente. Destes municípios, João Ramalho e Presidente Bernardes são de transmissão canina para leishmaniose visceral confirmada através de exames laboratoriais (sorológicos, parasitológicos e biologia molecular). Porém, Mirante do Paranapanema, está em investigação, aguardando resultados de exames de biologia molecular para identificação da espécie de Leishmania, até o fechamento desse estudo. 


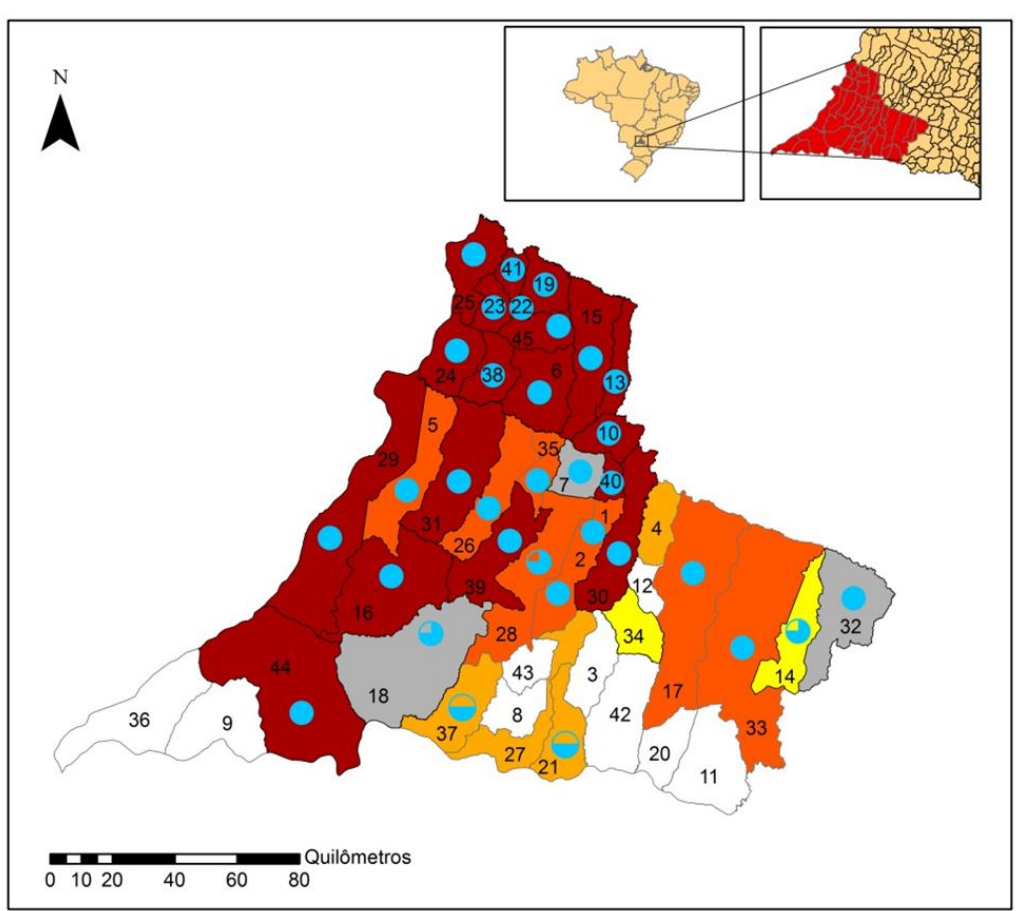

Legenda

Serviço de Zoonoses Totalmente Estruturado

- Serviços de Zoonoses Sem Estrutura Fisica, Parcialmente Funcionando.

Serviços de Zoonoses Sem Estrutura Fisica e Sem Funcionamento.

Municipio Em Investigação.

Municipio com Transmissão Humana e Canin Municipio com Transmissão Canina.

Municipio silencioso receptivo vulnerável

Municipio com notificação de casos caninos, mas não receptivo

Municipio silencioso não receptivo vulnerável

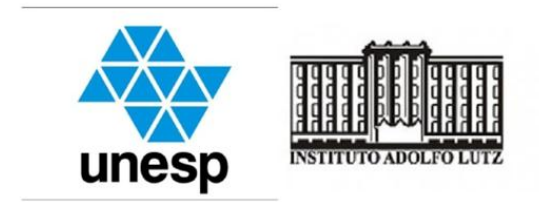

FONTES: Instituto Adolfo Lutz, IBGE. ESCALA: 1: 1.200.000.

ORG: MORENO, Matheus de Carvalho(2018). DATUM: SIRGAS 2000.

Figura 1. Situação epidemiológica de Leishmaniose Visceral e a disponibilidade de serviços de Zoonoses nos municípios da Região de Saúde de Presidente Prudente/SP/Brasil. Período Maio/2018.

Tabela 1. Classificação dos municípios da região de saúde de Presidente Prudente/SP, em relação ao Serviço de Zoonoses e situação epidemiológica de leishmaniose visceral em maio de 2018.

\begin{tabular}{lll}
\hline Municípios & Serviço de Zoonoses & Situação Epidemiológica \\
\hline 1. Alfredo Marcondes & Totalmente estruturado & Transmissão canina \\
2. Alvares Machado & Totalmente estruturado & Transmissão canina \\
3. Anhumas & Sem serviços zoonoses & Silencioso não receptivo vulnerável \\
4. Caiabu & Sem serviço zoonoses & Silencioso receptivo vulnerável \\
5. Caiuá & Totalmente estruturado & Transmissão canina \\
6. Dracena & Totalmente estruturado & Transmissão humana e canina \\
7. Emilianópolis & Totalmente estruturado & Em investigação \\
8. Estrela do Norte & Sem serviço zoonoses & Silencioso não receptivo vulnerável \\
9. Euclides da Cunha Paulista & Sem serviço zoonoses & Silencioso não receptivo vulnerável \\
10. Flora Rica & Totalmente estruturado & Transmissão humana e canina \\
11. lepê & Sem serviço zoonoses & Silencioso não receptivo vulnerável \\
12. Indiana & Sem serviço zoonoses & Silencioso não receptivo vulnerável \\
13. Irapuru & Totalmente estruturado & Transmissão humana e canina \\
14. João Ramalho & Sem estrutura física, parcialmente & Notificação de casos caninos / sem \\
15. Junqueirópolis & funcionando & presença de vetor \\
16. Marabá Paulista & Totalmente estruturado & Transmissão humana e canina \\
17. Martinópolis & Totalmente estruturado & Transmissão humana e canina \\
18. Mirante do Paranapanema & Totalmente estruturado & Transmissão canina
\end{tabular}

Colloq Vitae 2019 jan-abr; 11(1): 24-32. DOI: 10.5747/cv.2019.v11.n1.v249

ISSN 1984-6436/@ 2018 - Publicado pela Universidade do Oeste Paulista.

Artigo Open Access sob uma licença CC BY-NC-ND (http://creativecommons.org/licenses/by-nc-nd/4.0/). 


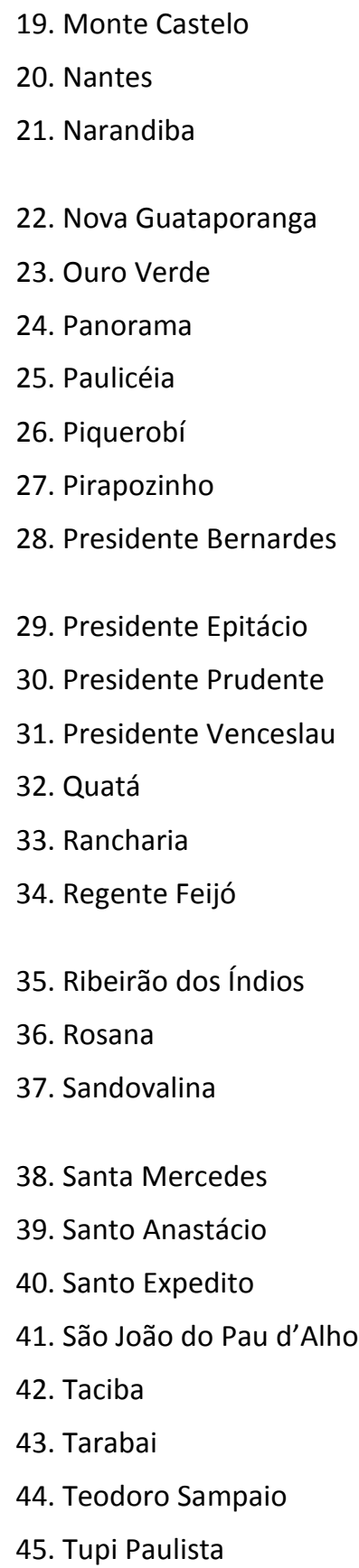

parcialmente funcionando

Totalmente estruturado

Sem serviço zoonoses

Sem estrutura física e sem

funcionamento

Totalmente estruturado

Totalmente estruturado

Totalmente estruturado

Totalmente estruturado

Totalmente estruturado

Sem serviço zoonoses

Sem estrutura física, parcialmente funcionando

Totalmente estruturado

Totalmente estruturado

Totalmente estruturado

Totalmente estruturado

Totalmente estruturado

Sem serviço zoonoses

Totalmente estruturado

Sem serviço zoonoses

Sem estrutura física e sem

funcionamento

Totalmente estruturado

Totalmente estruturado

Totalmente estruturado

Totalmente estruturado

Sem serviço zoonoses

Sem serviço zoonoses

Totalmente estruturado

Totalmente estruturado
Transmissão humana e canina

Silencioso não receptivo vulnerável

Silencioso receptivo vulnerável

Transmissão humana e canina

Transmissão humana e canina

Transmissão humana e canina

Transmissão humana e canina

Transmissão canina

Silencioso receptivo vulnerável

Transmissão canina

Transmissão humana e canina

Transmissão humana e canina

Transmissão humana e canina

Em investigação

Transmissão canina

Notificação de casos caninos / sem presença de vetor

Transmissão canina

Silencioso não receptivo vulnerável

Silencioso receptivo vulnerável

Transmissão humana e canina

Transmissão humana e canina

Transmissão humana e canina

Transmissão humana e canina

Silencioso não receptivo vulnerável

Silencioso não receptivo vulnerável

Transmissão humana e canina

Transmissão humana e canina

Fonte: Instituto Adolfo Lutz - Núcleo de Ciências Biomédicas de CRL Presidente Prudente.

Há 4,4\% (dois) municípios, Narandiba e Sandovalina que possuem equipe de Zoonoses capacitada pelo laboratório de referência regional, contudo, não é atuante. Não contam com estrutura física para realizar as atividades de inquérito sorológico (Tabela 1). Portanto, até a presente data, não realizam esse tipo de ação de vigilância recomendada pelo PVCLV. Ambos são municípios silenciosos receptivos vulneráveis, ou seja, não possuem casos de leishmaniose visceral notificados. Porém, existe a presença do vetor e o risco sanitário da ocorrência da doença.
Os municípios que não possuem Serviços de Zoonoses correspondem a 26,7\% (12), sendo eles: Anhumas, Caiabu, Estrela do Norte, Euclides da Cunha Paulista, lepê, Indiana, Nantes, Pirapozinho, Regente Feijó, Rosana, Taciba, Tarabai (Tabela 1). Em 20\% (nove) do total de municípios estudados: Anhumas, Estrela do Norte, Euclides da Cunha Paulista, lepê, Indiana, Nantes, Rosana, Taciba e Tarabai são classificados como silencioso não receptivo vulnerável, em que não há a presença do vetor. Em 2,2\% (um) município (Regente Feijó) possui notificação de 
casos caninos autóctones desde 2014, comprovados através de exames laboratoriais: sorologia, parasitológico e PCR positivos para leishmaniose visceral. Porém, até o momento não se tem registro do encontro do vetor pela Sucen de Presidente Prudente/SP. O referido município não tem Serviço de Zoonose e equipe estruturadas para realização de inquéritos sorológicos, mas realiza atividades de vigilância de animais suspeitos.

\section{DISCUSSÃO}

Ao analisar a disposição dos municípios que possuem transmissão de leishmaniose visceral humana e canina apresentados na Figura 1 , nota-se que eles estão próximos e/ou fazem fronteira. Analisando a situação epidemiológica da região, nota-se a que há uma relação de proximidade entre os municípios atingidos e que os mesmos seguem uma rota de dispersão da doença no sentido noroeste para sudeste. Isto é, da região da Alta Paulista que irradia para a região do Extremo Oeste, Pontal do Paranapanema, Alta Sorocabana a atinge também o Alto Capivari ${ }^{23}$. Esse grupo de municípios encontra-se localizado próximo à rodovia Marechal Rondon, que segundo Cardim e colaboradores, principal eixo de dispersão da doença e localizam-se próximos aos municípios de Ouro Verde e Dracena onde ocorreram os primeiros casos de leishmaniose visceral em $2005^{24,25}$. A expansão da doença pode ser influenciada pela circulação de produtos, pessoas, animais bens e mercadorias entre municípios que possui casos notificados ${ }^{23,24,26}$.

Dentre os municípios estudados e que não possuem Serviço de Zoonoses, os que se encontram em situação mais preocupante são: Regente Feijó, que mesmo classificado como não receptivo possui notificação de casos caninos; os municípios de Caiabu e Pirapozinho que são dois municípios que possuem o vetor desde 2011, mas que até o momento não realizaram nenhuma atividade de inquérito sorológico e de vigilância de animais suspeitos com leishmaniose visceral. Esses municípios apontados fazem fronteira com Presidente Prudente, que há transmissão humana e canina de leishmaniose visceral. Narandiba e Sandovalina, também merecem atenção especial, uma vez que ambos são silenciosos receptivos e que apesar de possuírem equipe capacitada, não possuem nenhuma estrutura física de Serviço de Zoonoses e também não realizam atividades de inquérito sorológico e busca ativa de casos suspeitos.

Dos municípios que possuem apenas transmissão canina, Presidente Bernardes ainda não possui um Serviço de Zoonoses com estrutura física. Portanto, o mesmo encontra-se parcialmente funcionando, uma vez que utiliza a estrutura física do CLR IAL PP V para realizar os testes de triagem sorológica para leishmaniose visceral canina. Já os demais municípios que possuem transmissão canina, o Serviço de Zoonoses encontra-se totalmente estruturado e funcionando o que favorece as ações de vigilância e controle. Este fato é importante, visto que na maioria dos casos, a transmissão da leishmaniose visceral canina precede a infecção no homem ${ }^{27,28}$.

No presente estudo, foram observados que os municípios de menor porte são justamente aqueles que enfrentam as maiores dificuldades, tanto na implantação dos Serviços de Zoonoses como na sua manutenção e execução contínua das ações de vigilância em saúde. Esse fato está relacionado diretamente com a situação socioeconômica dos municípios analisados. Dados similares foram observados em um estudo realizado por Gomes e colaboradores $^{29}$.

Outro ponto a ser levado em consideração nessa análise, é que a estruturação de Serviços de Zoonoses está ligada diretamente à condição epidemiológica de um determinado município. Uma vez que, a maioria deles que não o possuem, são municípios silenciosos receptivos e silenciosos não receptivos. Isto é, na maioria dos casos, o gestor só se preocupa com a estruturação do Serviço, frente a uma situação de risco eminente ou quanto há notificação de casos da doença em seu território e cobrança dos órgãos estaduais. Quando o ideal é que todos se estruturassem e realizassem atividades de vigilância com ações voltadas para prevenção e redução do risco de introdução da doença, uma vez que é recomendado pelo manual de vigilância, controle e prevenção de zoonose independente da classificação epidemiológica do município ${ }^{5}$.

A importância da continuidade das ações de vigilância e monitoramento da situação epidemiológica de uma determinada localidade foi evidenciada por um estudo realizado por Rangel e seus colboradores ${ }^{30}$, em que o município de Santo Expedito passou de Silencioso Receptivo Vulnerável (SRV) para município com transmissão 
humana no ano de 2013. Isso ocorreu por falta da existência de um Serviço de Zoonoses no município para identificar e notificar os casos de leishmaniose visceral canina, antes da ocorrência de casos humanos da doença.

Municípios que possuem Serviço de Zoonoses estruturado e atuante, o PVCLV recomenda que as ações realizadas necessitem ser avaliadas em relação à sua eficiência. Zuben e Donalisio relataram que nenhum dos municípios pesquisados realizava todas as atividades preconizadas pelo PVCLV e destacou como principais dificuldades de implantar o programa: a recusa da população a colaborar para o diagnóstico em cães, principalmente devido à eutanásia ser o principal método para o controle do reservatório doméstico ${ }^{21}$. Foi relatado ainda o alto custo do programa; falta de recursos financeiros e consequentemente recursos humanos e materiais, além da falta de comprometimento de envolvimento de gestores municipais ${ }^{21,31}$. Diante do exposto, mesmo aqueles municípios que possuem Serviço de Zoonoses e realizam todas as atividades preconizadas pelo PVCLV, podem não serem eficazes no controle da doença. É de grande importância a realização de estudos sobre a efetividade das ações realizadas, a fim de identificar aquelas que se mostraram mais

\section{REFERÊNCIAS}

1. Organização Mundial da Saúde (OMS). Zoonoses. 2017. Acesso em: 4 jul 2018. Disponível em: http://www.who.int/topics/zoonoses/en/

2. Acha PN, Szyfres B. Zoonosis y enfermedades transmisibles comunes al hombre $y$ a los animales. 3.ed. Washington: OPAS; 2001. 416p.

3. Miguel O. A vigilância sanitária e o controle das principais zoonoses. Epistéme. 1996;1(1):141-155.

4. Guimarães FF, Baptista AAS, Gustavo PMGM, Langoni $H$. Ações da vigilância epidemiológica e sanitária nos programas de controle de zoonoses. Rev Veterin Zoot. 2010;17(2):151-162.

5. Brasil. Ministério da Saúde. Secretaria de Vigilância em Saúde. Departamento de Vigilância das Doenças Transmissíveis. Manual de vigilância, prevenção e controle de zoonoses: normas técnicas e operacionais - Brasília: Ministério da Saúde. 2016; p121. eficientes e eficazes para uma determinada região.

\section{CONCLUSÕES}

É de grande relevância o papel que os Serviços de Zoonoses desempenham na vigilância em saúde não só para leishmaniose visceral, mas para outras doenças e que o gestor municipal priorize esse tipo de serviço. Sendo de extrema importância que os municípios o tenham disponíveis na escala local, que os mesmos sejam totalmente estruturados, funcionem com equipe capacitada e realizem ações constantes e eficazes de vigilância em saúde.

Há um número consideravelmente elevado de municípios que não possuem Serviço de Zoonoses totalmente estruturado, o que os torna vulneráveis em relação ao risco de ocorrência de leishmaniose visceral. Principalmente por pertencerem a uma região considerada endêmica para a doença, levando em consideração à sua localização, relação de proximidade e fluxo de mercadorias, pessoas, bens e serviços.

\section{CONFLITO DE INTERESSE}

Os autores declaram não haver qualquer potencial conflito de interesse que possa interferir na imparcialidade deste trabalho científico.

6. Rodrigues RCA, von Zuben APB, Lucca $T$, Castagna $C L$, Nitsche A, Presotto D, Kemp B et al. De CCZ a UVZ: mudança de paradigma no controle de zoonoses. BEPA. 2017;14(162):33-41.

7. Reichmann MLAB, Sandoval MRC, Formaggia DME, Presotto D, Nunes VFP, Santos LS et al. Orientação para projetos de centros de controle de zoonoses (CCZ - Manuais 2). São Paulo: Instituto Pasteur; 2004. p.45.

8. Gomes LH, Menezes RF. Diagnóstico de serviços de controle de zoonoses no Estado de São Paulo. BEPA. 2009;6(72):17-25.

9. Ministério da Saúde. Fundação Nacional de Saúde. Projetos físicos de unidades de controle de zoonoses e fatores biológicos de risco. Brasília: Funasa; 2003.

10. Brasil. Ministério da Saúde. Portaria no 1.138, de 23 de maio de 2014. Define as ações e os serviços de saúde voltados para vigilância, prevenção e controle de zoonoses e de acidentes causados por animais peçonhentos e venenosos, de relevância para a saúde pública. Brasília (DF), 2014. 
11. Brasil. Ministério da Saúde, Secretaria de Atenção à Saúde. Portaria no 758, de 26 de agosto de 2014. Inclui subtipo na Tabela de Tipos de Estabelecimentos de Saúde do SCNES. Diário Oficial da República Federativa do Brasil, Brasília (DF), 2014, ago 27, Seção 1:92.

12. Secretaria da Saúde, Coordenadoria de Controle de Doenças, Superintendência de Controle de Endemias. Manual de Vigilância e Controle da Leishmaniose Visceral Americana do Estado de São Paulo. São Paulo; 2006.

13. Brasil. Ministério da Saúde. Secretaria de Vigilância em Saúde. Coordenação-Geral de Desenvolvimento da Epidemiologia em Serviços. Guia de Vigilância em Saúde. 1.ed. Brasília: Ministério da Saúde; 2016. 773p.

14. Banuls AL, Hide M, Prugnolle F. Leishmania and the leishmaniases: a parasite genetic update and advances in taxonomy, epidemiology and pathogenicity in humans. Adv Parasitol. 2007;64:1-109. DOI: https://doi.org/10.1016/S0065-308X(06)64001-3

15. Alvar J, Vélez ID, Bern C, Herrero M, Desjeux $P$, Cano J et al. Leishmaniasis Worldwide and Global Estimates of Its Incidence. PLOS ONE. 2012;7(5):e35671. DOI: https://doi.org/10.1371/journal.pone.0035671

16. Organização Mundial da Saúde (OMS). Leishmaniose. 2014. Acesso em: 3 ago 2018. Disponível em: http://www.who.int/leishmaniasis/burden/en/

17. Souza MA, Nunes RFF, Viana TC, Marinho MJM, Moreira PVSQ, Pereira WO. Leishmaniose visceral humana: do diagnóstico ao tratamento. Rev Ciên Saúde Nov Esp. 2012;10(2):61-69.

18. Silva MRB. Avaliação da acurácia de testes imunocromatográficos rK39 no diagnóstico da leishmaniose visceral em pacientes coinfectados com HIV. [Tese de Doutorado] Goiânia: Universidade Federal de Goiás - UFG; 2014.

19. Lemos EM, Carvalho SFG, Dietze RC. Avaliação do teste rápido utilizando o antígeno recombinante $\mathrm{K} 39$ no diagnóstico da leishmaniose visceral no Brasil. Rev Soc Bras Med Tropical. 2003;36:36-38. DOI: http://dx.doi.org/10.1590/S0037-

$\underline{86822003000700011}$

20. Lindoso JAL, Goto $H$. Leishmaniose visceral: situação atual e perspectivas futuras. BEPA. 2006;3(26):7-11.

21. Zuben APB, Donalísio MR. Dificuldades na execução das diretrizes do Programa de Vigilância e Controle da Leishmaniose Visceral em grandes municípios brasileiros. Cad Saúde Púb. 2016;32:e00087415. DOI:

https://doi.org/10.1590/0102-311X00087415

22. Werneck GL. Controle da leishmaniose visceral no Brasil: o fim de um ciclo? Cad Saúde Púb. 2016;32(6):eED010616. DOI: http://dx.doi.org/10.1590/0102-311X00ED010616

23. D'Andrea LAZ. Leishmaniose visceral na região de Presidente Prudente, São Paulo: distribuição espacial e rotas de dispersão. [Tese de doutorado]. Pósgraduação em Geografia, Universidade Estadual Paulista, Presidente Prudente, 2017.

24. Cardim MFM, Rodas LAC, Dibo MR, Guirado MM, Oliveira AM, Chiaravalloti Neto $F$. Introdução e expansão da leishmaniose visceral americana em humanos no Estado de São Paulo, 1999-2011. Rev Saúde Púb. 2013;47(4):691-700. DOI: http://dx.doi.org/10.1590/S0034$\underline{8910.2013047004454}$

25. Cardim MFM, Guirado MM, Dibo MR, Neto FC. Leishmaniose visceral no estado de São Paulo, Brasil: análise espacial e espaço-temporal. Rev Saúde Púb. 2016;50:48. DOI: http://dx.doi.org/10.1590/S1518$\underline{8787.2016050005965}$

26. Mestre GLC, Fontes CJF. A expansão da epidemia da leishmaniose visceral no estado de Mato Grosso, 1998-2005. Rev Soc Bras Med Tropical. 2007;40(1):428. DOI: $\quad$ http://dx.doi.org/10.1590/S0037$\underline{86822007000100008}$

27. Borges LFNM, Lopes EGP, Freitas ACP, Silva MX, Haddad JPA, Silva JÁ, et. al. Prevalência e distribuição espacial da leishmaniose visceral em cães do município de Juatuba, Minas Gerais, Brasil. Ciên Rural. 2014;44(2):352-357.

DOI:

http://dx.doi.org/10.1590/S0103-

$\underline{84782014000200025}$

28. Oliveira CL, Assunção RM, Reis IA, Proietti FA. Spacial distribution of human and canine visceral leishmaniasis in Belo Horizonte, Minas Gerais State, Brazil, 1994-1997. Cad Saúde Púb. 2001;7(1):12311239. DOI: http://dx.doi.org/10.1590/S0102$\underline{311 \times 2001000500023}$

29. Gomes LH, Menezes RF, Aranda CMSS, Vieira PA. Serviços municipais de controle de zoonoses no Estado de São Paulo: diagnóstico situacional. BEPA. 2011;8(96):11-31.

30. Rangel O, Hiramoto RM, Henriques LDF, Taniguchi HH, Ciaravolo RMDC, Tolezano JE et al. Classificação epidemiológica dos municípios segundo o Programa de Vigilância e Controle da Leishmaniose Visceral 
Americana no estado de São Paulo, para 2013. BEPA. 2013;10(111):3-14.

31. D'Andrea, LAZ, Guimarães, RB. A importância da análise de distribuição espacial da leishmaniose visceral humana e canina para as ações de vigilância em saúde. Hygeia. 2018;14(28):121-138. DOI: http://dx.doi.org/10.14393/Hygeia142810

Recebido para publicação em 17/08/2018

Revisado em 24/08/2018

Aceito em 03/09/2018 